\title{
Uso de hábitats alterados por aves insectívoras de sotobosque en un gradiente ambiental y su potencial para la conservación en Nicoya, Costa Rica
}

\author{
Paul Eduardo Oviedo Pérez ${ }^{1}$ \\ 1. Universidad Estatal a Distancia, Maestría Académica en Manejo de Recursos Naturales, Cátedra Ecología y \\ Educación Ambiental, San José, Costa Rica; poviedop@uned.ac.cr
}

Recibido 13-VI-2019 • Corregido 5-X-2019 • Aceptado 11-XI-2019

DOI: https://doi.org/10.22458/urj.v12i1.2803

\begin{abstract}
Use of altered habitats by understory insectivore birds in an environmental gradient and their potential for conservation in Nicoya, Costa Rica". Introduction: Habitats disturbed by fragmentation and human activities increase the need to establish strategies to conserve species dependent on mature and continuous forests. Objective: To analyze the composition of insectivorous understory birds in three habitats with different degrees of forest disturbance, and to determine their conservation potential. Methods: I compared the composition of insectivorous understory birds with the mist netting traps. I characterized the habitats using the method of rectangular plots and based on the depth of the mulch, the luminosity, horizontal coverage of the vegetation of the understory, the forest basal area and the density of trees, palms and shrubs. Results: I captured a total of 397 individuals belonging to nine species. The mature forest was the habitat with the highest number of species (9) and individuals captured (139). Species composition varied significantly among habitats $\left(F_{(2}\right.$ 27) $=3,8758, p<0,001)$. The species with the greatest weight in the dissimilarity between the habitats were: Sittasomus griseicapillus, Thamnophilus doliatus and Eucometis penicillata. Disturbed habitats included more generalist species than the forest, dominated by specialist species. The tree and palm density best explained species association $\left(F_{(7,22)}=1,9774\right.$, rho $\left.=0,3794, p=0,003\right)$. There was no significant difference in the index of importance among the three habitats (ANOSIM, $p=0,63330$ ). Conclusion: Disturbed habitats are important for the conservation of some of the insectivorous bird species dependent on mature tropical forests in Nicoya.
\end{abstract}

Keywords: Tropical moist forest, composition, communities, conservation.
RESUMEN. Introducción: Los hábitats perturbados por la fragmentación y las actividades humanas aumentan la necesidad de establecer estrategias para conservar las especies que dependen de bosques maduros y continuos. Objetivo: Analizar la composición de aves insectívoras de sotobosque en tres hábitats con diferente grado de disturbio forestal y determinar su potencial de conservación. Métodos: Comparé la composición de aves con el método de captura con redes de niebla. Caractericé los hábitats utilizando el método de parcelas rectangulares y con base en la profundidad del mantillo, la luminosidad, la cobertura horizontal de la vegetación del sotobosque, el área basal del bosque y la densidad de árboles, palmeras y arbustos. Resultados: Capturé un total de 397 individuos pertenecientes a nueve especies. El bosque maduro fue el hábitat con mayor cantidad de especies (9) y de individuos capturados (139). La composición de especies varió significativamente entre los hábitats $\left(F_{(2,27)}=3,87 ; p<0,001\right)$. Las especies con mayor peso en la disimilitud entre los hábitats fueron: Sittasomus griseicapillus, Thamnophilus doliatus y Eucometis penicillata. El ensamblaje de las especies en los hábitats más perturbados incluyó especies más generalistas en comparación con el bosque maduro, donde hubo mayor abundancia de especies especialistas. La densidad arbórea y de palmas fueron los atributos del hábitat que mejor explicaron la asociación de las especies $\left(F_{(7,22)}=1,98 ; r h o=0,379 ; p=0,003\right)$. No hubo diferencia significativa en el índice de importancia entre los tres hábitats (ANOSIM, $p=0,633$ ). Conclusión: Los hábitats perturbados son importantes para la conservación de algunas de las especies de aves insectívoras dependientes de bosques maduros tropicales.

Palabras clave: Bosque húmedo tropical, composición, comunidades, conservación. 
Las principales amenazas para la conservación de la biodiversidad en los bosques neotropicales son la fragmentación y la destrucción de los hábitats (Pimm \& Raven, 2000). Varias décadas de deforestación causada por la actividad agropecuaria, la extracción maderera y el crecimiento de la población humana han reducido el bosque a remanentes aislados, que varían en su grado de heterogeneidad vegetal y similitud con el bosque original (Cerezo, Robbins, \& Dowell, 2009; Meave, Romero-Romero, Salas-Morales, Pérez-García, \& Gallardo-Cruz, 2012).

Aproximadamente unas 283 213ha $(5,5 \%)$ del territorio de Costa Rica están cubiertas por bosque húmedo tropical (Quesada, 2007). Aunque buena parte de este bosque está protegido por alguna categoría de conservación, existen áreas de vegetación secundaria inmersas dentro de una matriz agropecuaria (Sáenz, Villatoro, Ibrahim, Fajardo, \& Pérez, 2006). Esto ha motivado a los ecólogos del paisaje a realizar estudios para comprender el potencial de estos remanentes para la conservación de las especies (Harvey et al., 2006). Los estudios realizados en los fragmentos de bosque han demostrado la importancia de los hábitats modificados para la conservación de aves, tanto migratorias como residentes neotropicales de borde de bosque (Martínez-Salinas \& DeClerck, 2010).

La manera en que la alteración del hábitat afecta la comunidad de aves de bosque es aún tema de debate. Por un lado, algunos estudios indican que los disturbios sobre la vegetación disminuyen tanto la cantidad de individuos como el número de especies dependientes de bosque maduro (Rappole \& Morton, 1985; Sekercioglu, 2002; Cárdenas, Harvey, Ibrahim, \& Finegan, 2003). Por el contrario, otras investigaciones sugieren que los continuos cambios en la matriz del ecosistema han promovido que las aves neotropicales deban adaptarse, utilizando recursos alimentarios y refugios no habituales en los hábitats alterados (Daily, Ehrlich, \& Sánchez-Azofeifa, 2001; Cepeda \& Montero, 2011; Villegas \& Villalobos, 2014). Una parte de la avifauna que aún sobrevive en paisajes alterados corresponde a especies insectívoras de sotobosque. Las aves que pertenecen a este gremio están más confinadas al interior de bosque debido a su alta especificidad de hábitat y baja movilidad (Kricher, 1999; Idrobo-Medina \& Gallo-Cajiao, 2012).

La importancia de la cobertura boscosa y la conectividad para la conservación de la biodiversidad en zonas alteradas por la ganadería ha sido estudiada en toda la región Mesoamericana (Harvey et al., 2006) y en diferentes zonas de Sudamérica (Naranjo \& Chacón, 1997). Por ejemplo, un estudio realizado en Chiapas, México, demostró que la degradación y la fragmentación de las zonas boscosas afecta más a las especies del interior del bosque, que por lo general no están adaptadas a cruzar áreas abiertas (Rangel-Salazar, Enríquez, \& Sántiz, 2009). Asimismo, Cerezo et al. (2009), concluyen que la eliminación y la fragmentación de bosques tropicales en Guatemala son la causa de la desaparición de la mayoría de las especies dependientes de bosque. En Costa Rica se ha podido demostrar que en fragmentos de bosque tropical ubicados en la región de Sarapiquí, el índice de área foliar y el porcentaje de apertura del dosel afectan de manera diferente la abundancia de tres aves insectívoras de sotobosque (Sánchez-Ulate, 2011). En la Península de Nicoya de Costa Rica, todavía existe un vacío de información sobre la importancia de los hábitats alterados por la ganadería para la conservación de fauna típica de bosque. El objetivo de este estudio fue analizar los cambios en la composición y abundancia de aves insectívoras de sotobosque en un gradiente ambiental dentro de un paisaje ganadero; así como, determinar el potencial de conservación de los hábitats alterados de esa región para este gremio de aves. La hipótesis es que las condiciones del fragmento se correlacionan con el número de especies de la biota original. 


\section{MATERIALES Y MÉTODOS}

Área de estudio: El estudio fue realizado en tres sitios: Reserva Nacional Absoluta Cabo Blanco (RNACB), zona de amortiguamiento de esta área y el bosque ribereño del río Lajas, al suroeste de la Península de Nicoya, Costa Rica, entre las coordenadas $\left(9^{\circ} 33^{\prime} 0^{\prime \prime}-9^{\circ} 36^{\prime} 0^{\prime \prime} \mathrm{N} \& 85^{\circ} 05^{\prime} 0^{\prime \prime}\right.$ $85^{\circ} 09^{\prime} 0^{\prime \prime} \mathrm{W}$ ). El clima de la zona es cálido, con una estación seca bien definida entre los meses de diciembre a abril. La precipitación oscila entre 2500 - 3 200mm anuales, la temperatura media anual es de $27^{\circ} \mathrm{C}$ y la humedad relativa promedio anual es del $81 \%$ (Jiménez, 2013). La zona de vida es bosque muy húmedo premontano transición a basal (bmh-P) (Holdridge, 1970).

Descripción de los hábitats: El interior de bosque de la RNACB (a partir de ahora bosque maduro) se caracterizó por presentar tres estratos de vegetación. El dosel estuvo dominado por árboles de Pseudobombax septenatum, Spondias mombin y Anacardium excelsum. El sotobosque incluyó arbustos de varias especies de Rubiaceae, árboles jóvenes del género Trichilia, palma real (Attalea rostrata) y lianas del género Bahunia (Camacho \& Stewart, 2007). Este hábitat tiene una extensión de 1 172ha y ha permanecido protegido por más de 30 años (Boza \& Mendoza, 1980).

La zona de amortiguamiento consistió en cinco remanentes de vegetación secundaria (a partir de ahora bosque secundario), cuya superficie varió entre 5 y 17 ha y que fueron colindantes con el límite sureste de la RNACB. El dosel estuvo dominado por fustales de Enterolobium cyclocarpum y Samanea saman. El sotobosque fue denso con abundante palma real (A. rostrata) y especies de árboles pioneros, principalmente Sapium glandulossum y Stemmadenia donnell-smithii (Oviedo, 2015). La ganadería de subsistencia, viviendas aisladas, calles de lastre y extracción selectiva de madera fueron los principales disturbios observados en este hábitat.

La franja de vegetación en ambos bordes del río Lajas (a partir de ahora bosque ribereño) osciló entre 10 y $35 \mathrm{~m}$ y estuvo rodeada por lotes de pastura de varias fincas ganaderas. El estrato arbóreo estuvo dominado por Samanea saman y Bombacopsis quinata. El sotobosque tuvo una alta abundancia de Guazuma ulmifolia y varias especies de leguminosas (Oviedo, 2015). Este hábitat estuvo localizado a $5 \mathrm{~km}$ de la RNACB y es considerado como el más alterado, tanto por la falta de conectividad como por la actividad ganadera a gran escala.

Variables ambientales: Para la caracterización de los hábitats utilicé el método de parcelas de 10mx30m (Gysel \& Lyon, 1990), diez parcelas por hábitat separadas entre sí por una distancia de $100 \mathrm{~m}$. El muestreo fue realizado de mayo a julio (época lluviosa) y rotando las parcelas entre los hábitats. Tanto en las esquinas de cada parcela como en el centro, estimé la luminosidad del sotobosque (lux) con un medidor de luz digital Extech 401025 y la profundidad del mantillo, introduciendo una regla metálica graduada en centímetros. La luminosidad fue medida entre las 10:00 y las 11:30a.m. Además, estimé la cobertura horizontal de la vegetación del sotobosque (CHVS), por medio del conteo de barras blancas de $10 \mathrm{~cm}$ de ancho trazadas sobre un tubo de PVC. Esta lectura la realicé a una distancia de $5 \mathrm{~m}$ del tubo y en dirección de los cuatro puntos cardinales. La lectura fue clasificada como un $0 \%$ de cobertura cuando visualicé las diez barras y un $100 \%$ de cobertura cuando ninguna barra fue observada. Estimé el área basal ( $\left.\mathrm{m}^{2} / \mathrm{ha}\right)$ de los árboles que a una altura de $1,3 \mathrm{~m}$ sobre el suelo tuvieran un diámetro (DAP) mayor o igual a $10 \mathrm{~cm}$ dentro de cada parcela. La densidad arbórea (DA), arbustiva (DB) y de palmas (DP) por parcela fue estimada como la cantidad de individuos por hectárea.

Muestreo de aves: Para efectos de este estudio definí como aves insectívoras de sotobosque, a aquellas especies que se alimentan principalmente de insectos atrapados en la superficie de la corteza de los árboles, en el suelo y en el follaje, según la clasificación propuesta por González-Salazar, Martínez-Meyer y López-Santiago (2014). Las comunidades de aves insectívoras de sotobosque presentes en los tres hábitats fueron determinadas utilizando el método de capturarecaptura con redes de niebla (Ralph et al., 1996). A partir del centro de cada parcela, coloqué seis 
redes de niebla $(2,5 \mathrm{~m}$ de altura, $12 \mathrm{~m}$ de longitud de malla de $32 \mathrm{~mm})$, separadas entre sí por una distancia mínima de 15m. El muestreo se realizó de enero a abril del 2017 (época seca) y de junio a agosto del mismo año (época lluviosa). Las redes fueron rotadas entre los hábitats a lo largo del periodo de estudio. Las redes estuvieron abiertas desde el ras del suelo hasta una altura de $2 \mathrm{~m}$. La captura de aves fue realizada de 07:00 a 11:00a.m. y de 15:00 a 17:00p.m. Un día de muestreo correspondió a 36/h/red. En total cada hábitat fue muestreado 10 días distribuidos a lo largo del periodo de estudio. El esfuerzo total de captura de aves fue de $1080 \mathrm{~h} / \mathrm{red}$ (360h/red por hábitat). Las aves capturadas fueron anilladas para evitar el doble conteo de individuos.

Análisis de datos: Los datos obtenidos para caracterizar cada uno de los hábitats (lux, mantillo, CHVS, área basal, DA, DB y DP) fueron ordenados en una matriz de variables ambientales. Asimismo, la lista de especies capturadas y sus respectivas abundancias fueron ordenadas en una matriz de comunidad. Excluí de los análisis estadísticos las capturas de especies neárticas y aquellas cuya alimentación no incluyera artrópodos, utilizando como referencia la dieta documentada por Stiles y Skutch (2003).

La comparación de la composición específica entre hábitats fue realizada con la rutina no paramétrica ANOSIM ("analysys of similarities"), bajo la hipótesis nula de que no hay diferencia en la composición de especies de aves insectívoras de sotobosque entre los tres hábitats. ANOSIM trabaja como una prueba de ANOVA, donde en lugar de operar sobre datos sin procesar, opera en una matriz de disimilitud clasificada (Clarke, 1993). Para evitar que las especies más comunes dominaran en el resultado final de la ordenación y para aumentar la influencia de las especies subordinadas en el modelo resultante, las abundancias fueron transformadas por medio de la función decostand y el método Hellinger del paquete estadístico vegan (Oksanen et al., 2013). Para visualizar el agrupamiento de las especies de aves entre los hábitats con base a su riqueza y abundancia, utilicé el escalamiento multidimensional no métrico (nMDS) y el índice de Bray-Curtis (Herrando-Pérez, 2002; Calderón, Galindo, \& Cedeño, 2008), por medio del paquete estadístico vegan y ggplot2 (Oksanen, 2015), que producen diagramas bidimensionales de relaciones entre ubicaciones. La contribución de las especies a la diferenciación o similitud entre grupos fue analizada con el módulo SIMPER ("similarity profile"). Las correlaciones entre la abundancia relativa por especie y los variables ambientales fueron estimadas a través del análisis de redundancia canónica (RDA) y coeficiente de correlación de Spearman (rho), con el respectivo cálculo de su significancia estadística por medio de un ANOVA (Escudero \& Rubio, 1994). Previo a este análisis, todos los valores ambientales fueron estandarizados por medio de la función decostand y el método standardize del paquete estadístico vegan.

La importancia relativa $(I)$ de los hábitats para la conservación de las especies de bosque fue estimada por medio del índice desarrollado por Petit y Petit (2003), que consiste en un puntaje acumulativo basado en la fórmula:

$$
I j \sum_{i=1}^{n} V i P i j
$$

donde;

$V_{i}$ es un puntaje de vulnerabilidad para cada especie, que representa el atributo "sensibilidad a la perturbación" (baja=1, media=2, alta=3) establecido por Stotz, Fitzpatrick, Parker y Moskovits (1996); $n$ es la cantidad de especies y $P_{i j}$ representa la proporción de la especie $i$ detectada en el hábitat $j$. Por lo tanto, el valor de $\mathrm{P}_{i j}$ para cada especie varía entre 0 y 1 dentro del hábitat y suma 1 entre todos los hábitats (Cerezo et al., 2009). 
Ética, conflicto de intereses y declaración de financiamiento: El autor declara haber cumplido con todos los requisitos éticos y legales pertinentes, tanto durante el estudio como en el manuscrito; que no hay conflictos de interés de ningún tipo, y que todas las fuentes financieras se detallan plena y claramente en la sección de agradecimientos. Asimismo, está de acuerdo con la versión editada final del documento. El respectivo documento legal firmado se encuentra en los archivos de la revista.

\section{RESULTADOS}

Un total de 397 individuos clasificados en nueve especies de aves insectívoras de sotobosque fueron capturados. La tasa media de captura fue de $0,42 \pm 0,086 a v e s / r e d / h$. El hábitat con más individuos capturados fue el bosque secundario. Por el contrario, el bosque ribereño fue el hábitat con menor cantidad de especies y de individuos capturados (Cuadro 1).

\section{CUADRO 1}

Riqueza y abundancia de aves insectívoras de sotobosque en tres hábitats con diferente grado de perturbación, suroeste de la Península de Nicoya, Costa Rica

\begin{tabular}{cccc}
\hline Variable & Hóbitat & \\
& Bosque maduro & Bosque secundario & Bosque ribereño \\
\hline Riqueza de especies & 9 & 9 & 5 \\
Abundancia total & 139 & 168 & 90 \\
Tasa media de captura (aves/red/h) & $0,4 \pm 0,083$ & $0,51 \pm 0,023$ & $0,34 \pm 0,17$ \\
\hline
\end{tabular}

El análisis de varianza aplicado (ANOSIM) mostró que la composición de la comunidad de aves insectívoras de sotobosque varió significativamente entre los tres hábitats $\left(F_{(2,27)}=3,88\right.$; $\mathrm{p}<0,001$ ). El análisis multidimensional no métrico (nMDS) separó a las especies de aves en dos grupos. El primer grupo (izquierda del gráfico) estuvo constituido por cuatro especies aves capturadas principalmente en las parcelas de bosque maduro (Sittasomus griseicapillus, Platyrinchus cancrominus, Dendrocincla homochroa y Xyphorynchus flavigaster). El segundo grupo (derecha del gráfico) estuvo compuesto por otras cuatro especies asociadas principalmente a los hábitats más alterados (Fig. 1).

Los análisis SIMPER mostraron que las especies S. griseicapillus, Thamnophilus doliatus y Eucometis penicillata fueron responsables de una alta contribución en la disimilitud de la composición entre los hábitats analizados. Esas tres especies y Thryophilus rufalbus contribuyeron con más del $50 \%$ de la disimilitud de la composición entre el bosque maduro y el bosque secundario. Asimismo, las primeras tres especies anteriormente mencionadas, junto al piquichato norteño ( $P$. cancrominus), aportaron más del $60 \%$ de la disimilitud de la composición entre el bosque maduro y el bosque ribereño (Cuadro 2). Dos individuos de la especie $E$. penicillata anillados en el bosque maduro fueron recapturados en el bosque ribereño. 


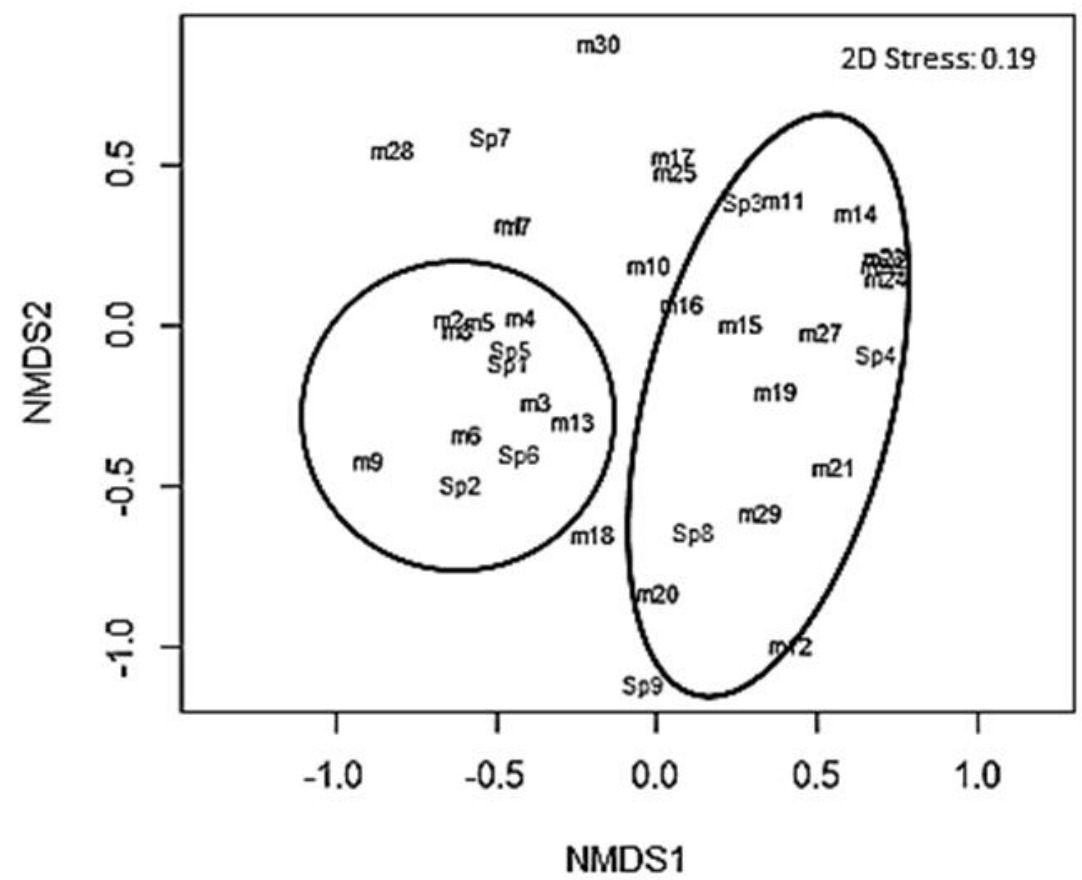

Fig. 1: Disimilitud entre uso de hábitat y especies de aves insectívoras asociadas, mediante análisis multidimensional no métrico (nMDS). La letra " $m$ " representa la unidad de muestra (bosque maduro $=m_{1}-m_{10}$, bosque secundario $m_{11}-m_{20}$, bosque ribereño $\left.=m_{21}-m_{30}\right)$. $S p_{i}$ indica el código asignado a la especie $i$.

\section{CUADRO 2}

Contribución de las especies a las diferencias (disimilitud) entre hábitats basados en análisis SIMPER. (1) bosque maduro, (2) bosque secundario y (3) bosque ribereño

\begin{tabular}{|c|c|c|c|c|c|c|c|}
\hline \multirow[t]{2}{*}{ Especie } & \multirow[t]{2}{*}{ Código } & \multicolumn{3}{|c|}{ Media } & \multicolumn{3}{|c|}{$\begin{array}{l}\text { Contribución media de cada } \\
\text { especie entre hábitats }\end{array}$} \\
\hline & & $\begin{array}{l}\text { Hábitat } \\
(1-2)\end{array}$ & $\begin{array}{l}\text { Hábitat } \\
(1-3)\end{array}$ & $\begin{array}{l}\text { Hábitat } \\
(2-3)\end{array}$ & $\begin{array}{l}1 \text { vs } 2 \\
(58 \%)\end{array}$ & $\begin{array}{l}1 \text { vs } 3 \\
(64 \%)\end{array}$ & $\begin{array}{l}2 \text { vs } 3 \\
(66 \%)\end{array}$ \\
\hline Sittasomusgriseicapillus & Sp1 & 0,1221 & 0,1560 & 0,0569 & 0,1819 & 0,2126 & 0,6611 \\
\hline Platyrinchuscancrominus & $\mathrm{Sp} 2$ & 0,0808 & 0,0981 & 0,0407 & & 0,6471 & \\
\hline Thryophilusrufalbus & Sp3 & 0,0847 & 0,0862 & 0,1183 & 0,5842 & & 0,1967 \\
\hline Thamnophilusdoliatus & Sp4 & 0,0954 & 0,1125 & 0,1165 & 0,3241 & 0,3661 & 0,3905 \\
\hline Dendrocinclahomochroa & Sp5 & 0,0735 & 0,0929 & 0,0390 & & & \\
\hline Xiphorhynchusflavigaster & Sp6 & 0,0452 & 0,0429 & 0,0248 & & & \\
\hline Eucometispenicillata & Sp7 & 0,0899 & 0,1080 & 0,1058 & 0,4580 & 0,5133 & 0,5664 \\
\hline Attilaspadiceus & Sp8 & 0,0414 & 0,0240 & 0,0537 & & & \\
\hline Basileuterusrufifrons & Sp9 & 0,0381 & 0,0128 & 0,0454 & & & \\
\hline
\end{tabular}

El análisis de ordenación mediante el RDA extrajo un eje I marcado por la densidad arbórea y un eje II marcado por la densidad de palmas (Fig. 2). Ambas variables ambientales explicaron de forma significativa el ensamblaje de las especies de aves insectívoras $\left(F_{(7,22)}=1,9774\right.$; rho $=0,3794$; $p=0,003)$. No obstante, el análisis RDA indica que la totalidad de las variables ambientales utilizadas en este modelo, sólo permiten explicar un $38 \%$ de las tendencias de agrupación de las especies. 


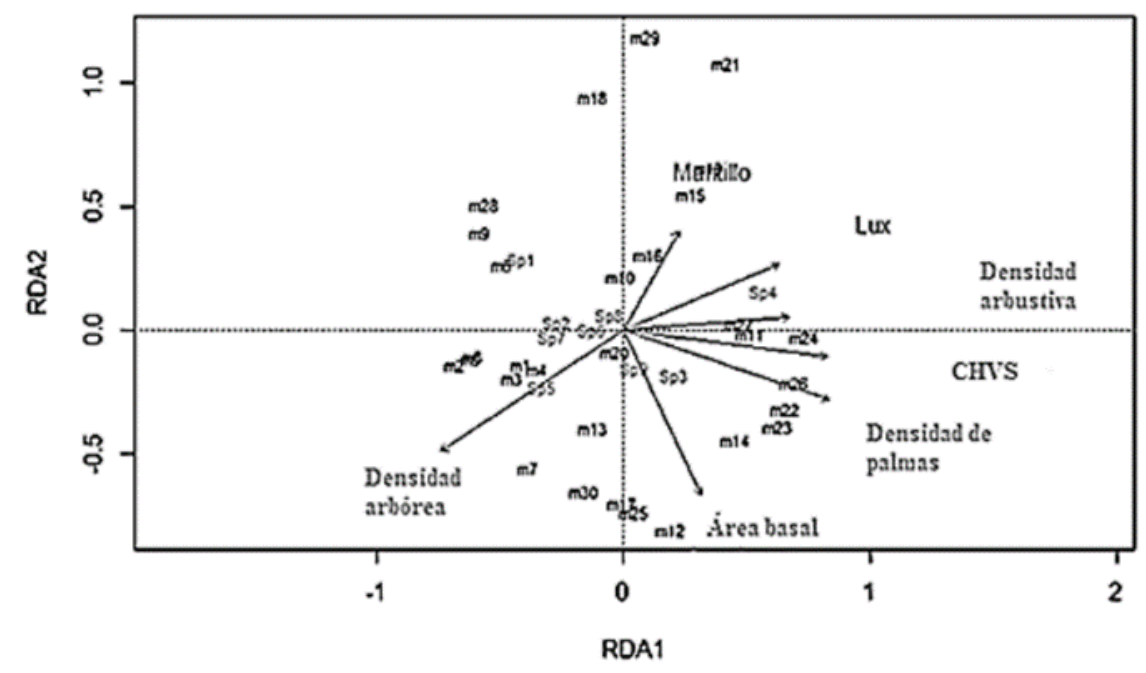

Fig. 2. Ordenación con los ejes I y II del RDA utilizando como matriz explicativa siete variables ambientales (densidad arbórea, profundidad del mantillo, luminosidad (lux), densidad arbustiva, cobertura horizontal de la vegetación del sotobosque (CHVS), densidad de palmas y área basal), con la situación de las parcelas $\left(\mathrm{m}_{i}\right)$ y las especies de aves insectívoras $\left(\mathrm{Sp}_{i}\right)$.

No se encontraron diferencias significativas en el índice de importancia entre los tres hábitats (ANOSIM, $\mathrm{p}=0,63330$ ). Solamente el trepador rojizo ( $D$. homochroa) mostró un valor alto de sensibilidad a la perturbación. Por otra parte, las tres especies de la familia Dendrocolaptidae ( $D$. homochroa, S. greiseicapillus y $X$. flavigaster) disminuyeron su abundancia a medida que se alejaba del bosque maduro, hasta estar ausentes en el hábitat más alterado (bosque ribereño). Las especies que mostraron estar más afiliadas al hábitat altamente perturbado fueron el batará barreteado ( $T$. doliatus) y el soterrey rufo y blanco (T. rufalbus) (Cuadro 3).

\section{CUADRO 3}

Especies de aves registradas y sus respectivas abundancias relativas medias. Tipos de hábitat: Bm (bosque maduro), Bs (bosque secundario en zona de amortiguamiento) y $\mathrm{Br}$ (bosque ribereño). Sensibilidad a la perturbación: B (baja), M (media) y A (Alta)

\begin{tabular}{|c|c|c|c|c|c|c|c|}
\hline \multirow[t]{2}{*}{ Familia/especie } & \multirow{2}{*}{$\begin{array}{c}\text { Abundancia } \\
\text { relativa } \\
\text { (aves/h/red) }\end{array}$} & \multicolumn{3}{|c|}{ Hábitat } & \multicolumn{3}{|c|}{$\begin{array}{l}\text { Sensibilidad a la } \\
\text { perturbación }\end{array}$} \\
\hline & & $\mathrm{Bm}$ & Bs & $\mathrm{Br}$ & B & M & A \\
\hline \multicolumn{8}{|l|}{ Dendrocolaptidae } \\
\hline Dendrocincla homochroa & 0,5 & $x$ & $x$ & & & & $x$ \\
\hline Sittasomus griseicapillus & 1 & $x$ & $x$ & & & $x$ & \\
\hline Xiphorhynchus lachrymosus & 0,23 & $x$ & $x$ & & & $x$ & \\
\hline \multicolumn{8}{|l|}{ Thamnophilidae } \\
\hline Thamnophilus doliatus & 1 & $x$ & $x$ & $x$ & $x$ & & \\
\hline \multicolumn{8}{|l|}{ Tyrannidae } \\
\hline Attila spadiceus & 0,27 & $x$ & $x$ & $x$ & $x$ & & \\
\hline Platyrinchus cancrominus & 0,53 & & & & & $x$ & \\
\hline \multicolumn{8}{|l|}{ Troglodytidae } \\
\hline Thryophilus rufalbus & 1,17 & $x$ & $x$ & $x$ & & $x$ & \\
\hline \multicolumn{8}{|l|}{ Parulidae } \\
\hline Basileuterus rufifrons & 0,2 & $x$ & $x$ & & $x$ & & \\
\hline \multicolumn{8}{|l|}{ Thraupidae } \\
\hline Eucometis penicillata & 0,87 & $x$ & $x$ & $x$ & & $x$ & \\
\hline
\end{tabular}




\section{DISCUSIÓN}

Existen diferentes estudios que indican la influencia de la fragmentación y la alteración del hábitat sobre la disminución, tanto en la riqueza como en la abundancia, de aves insectívoras en bosques tropicales (Canterbury, Martin, Petit, Petit, \& Bradford, 2000; Marini, 2001; Sekercioglu et al., 2002; Idropo-Medina \& Gallo-Cajiao, 2012); pero aún no se han encontrado estudios que describan con exactitud cuáles atributos del hábitat son los que mejor explican los patrones de organización de las especies que pertenecen a este gremio. Según Sanchéz-Ulate (2011), el área del fragmento, el índice de área foliar y la abundancia de artrópodos, son atributos de hábitat que están relacionados positivamente con la presencia de aves insectívoras de sotobosque. Aunque la actividad ganadera modifica el microclima local, originando condiciones ambientales más secas y áridas, tanto en las pasturas como en la vegetación circundante (Lambin, Geits, \& Lepers, 2003), los resultados de este estudio muestran que una porción de la avifauna insectívora de sotobosque que persiste en los hábitats alterados y rodeados por fincas ganaderas es comparable con la del bosque maduro original. Por otra parte, los bosques secundarios de la zona de amortiguamiento ofrecen hábitat para una parte importante de aves insectívoras y pueden sustentar más individuos que el bosque maduro. Lo anterior es debido a que los bordes de bosque ofrecen una mayor cantidad de nichos en comparación con los bosques maduros (MacArthur \& Wilson, 1967; Verea \& Solorzano, 1998). Asimismo, se ha demostrado que los recursos alimentarios (e.g. poblaciones de insectos) son menos estacionales en estos ecotonos (Loiselle, 1988; Naranjo \& Chacón, 1997).

Es posible que tanto la riqueza como la abundancia de aves insectívoras hayan sido subestimadas en el bosque ribereño, debido a la alta actividad de otros gremios de aves que fueron capturadas en ese hábitat. La presencia de personas cerca de las redes liberando a las aves no insectívoras pudo provocar un disturbio que alejara a las especies propias de este estudio, cosa que no sucedió en el bosque maduro y bosque secundario, hábitats donde la mayoría de aves capturadas fueron insectívoras. La disminución de trepatroncos (Dendrocolaptidae) en el hábitat más alterado (bosque ribereño) puede ser explicada por la alta especificidad de hábitat y baja movilidad que caracteriza a las especies de esta familia. Por consiguiente, las especies de trepatroncos forman parte del $60 \%$ de las especies residentes de aves terrestres que requieren grandes áreas boscosas, o fragmentos de bosque interconectados, para sobrevivir (Stiles, 1985; Stratford \& Stouffer, 1999; Sekercioglo et al., 2002). Por el contrario, una mayor abundancia de $T$. doliatus y $T$. rufalbus en el bosque secundario y ribereño se debe a sus hábitos generalistas (Stotz et al., 1996; Stiles \& Skutch, 2003); es decir, que fueron capturadas en los tres hábitats, pero en mayor cantidad en los hábitats alterados. La recaptura de dos individuos de la tangara cabecigris (E. penicillata) a más de $5 \mathrm{~km}$ del sitio donde fueron anillados, demuestra que es una especie con alta movilidad en el paisaje. Debido a que todas las especies capturadas en el bosque ribereño estuvieron también presentes en el bosque maduro, se puede concluir que los hábitats alterados tienen algún valor para un subconjunto de aves, especialmente para aquellas más generalistas en uso de hábitat (Cerezo et al., 2009).

Los resultados de este estudio muestran que ante especies consideradas raras o de baja abundancia, es recomendable analizar el uso de hábitat a partir de la composición y no exclusivamente por la riqueza y la abundancia relativa de las especies. De considerarse sólo estos dos parámetros ecológicos, sin considerar la composición y sin discriminar su grado de especificidad de hábitat, se podría llegar a concluir que los hábitats con diferente grado de disturbio tienen la misma importancia para la conservación de las aves insectívoras de sotobosque. Asimismo, haber tenido un valor más bajo de importancia para la conservación en el bosque ribereño, aunque sea no significativo, indica que este hábitat sustenta parte importante de la biota original, aunque con poco valor de conservación, criterio compartido con Petit y Petit (2003). 
La fisonomía del hábitat ha sido considerada por varias décadas como una importante dimensión del nicho para la avifauna, debido a que amortigua las tensiones fisiológicas; además, provee protección contra depredadores, sustratos de anidación y diversidad de presas (Weins \& Rotenberry, 1981; Naranjo \& Chacón, 1997; Luck \& Daily, 2003; Rangel-Salazar, Enríquez, \& Sántiz, 2009; Leighton, Mendenhall, Rosales, Zahawi, \& Holl, 2014). Los resultados de este estudio sugieren que la densidad arbórea y de palmas son aspectos estructurales de la vegetación correlacionados positivamente con la ocupación del hábitat y la abundancia de especies de aves insectívoras de sotobosque. Sin embargo, todavía la mayoría de variables ambientales que explican la organización de las comunidades aviares deben ser investigadas.

La información obtenida en este estudio permitió determinar que la alteración del hábitat por las actividades socioeconómicas provoca cambios en la composición de las aves insectívoras; así como, que parte de la biota original persiste en hábitats alterados. La densidad arbórea juega un papel fundamental entre los atributos de hábitat para la conservación de la avifauna. La protección y expansión del bosque ribereño debe ser incentivada para prevenir las extinciones locales. Investigaciones futuras deberán incorporar dentro de las variables ambientales el tamaño de hábitat y la estimación de presas potenciales para las aves insectívoras. Además, se hace un llamado a la comunidad científica sobre la importancia de analizar las comunidades de aves en función de la composición de especies y su grado de especificidad (generalistas y especialistas); especialmente cuando se trabaje con especies que por naturaleza son consideradas como raras o poco abundantes.

\section{AGRADECIMIENTOS}

Agradezco a Michael McCoy, Rose Marie Menacho, Paola Gastezzi y Zaidett Barrientos por la revisión científica del manuscrito. A Meyer Guevara por el apoyo en los análisis estadísticos. A Natalia Corrales y Andrés Jiménez por la colaboración en la toma de datos de campo. Este trabajo fue realizado con el apoyo de Idea Wild, Association of Field Ornithologists y el Sistema Nacional de Áreas de Conservación (SINAC). Este trabajo es parte de los requisitos de graduación del programa de Maestría Académica en Manejo de Recursos Naturales de la Universidad Estatal a Distancia de Costa Rica.

\section{REFERENCIAS}

Boza, M. \& R. Mendoza. (1980). Los parques nacionales de Costa Rica. Madrid, España: INCAFO.

Calderón, R. R., Galindo, C., \& Cedeño, J. R. (2008). Utilización de hábitat por reptiles en estados sucesionales de selvas tropicales de Campeche, México. Acta Zoológica Mexicana, 24(1), 95-114. DOI: 10.21829/azm.2008.241626

Camacho, F., \& Stewart, E. (2007). Árboles comunes de la Reserva Natural Absoluta Cabo Blanco. Heredia, Costa Rica: INBio.

Canterbury, G., Martin, T., Petit, D., Petit, L., \& Bradford, D. (2000). Bird communities and habitat as ecological indicators of forests condition in regional monitoring. Conservation Biology, 14(2), 544-558. DOI: 10.1046/j.15231739.2000.98235.x

Cárdenas, G., Harvey, C., Ibrahim, M., \& Finegan, B. (2003). Diversidad y riqueza de aves en diferentes hábitats en un paisaje fragmentado en Cañas, Costa Rica. Agroforestería en las Américas, 10(39-40), 78-85.

Cepeda, M. F., \& Montero, J. L. (2011). Variación en la composición y abundancia de aves terrestres en la Reserva de la Biosfera Ría Lagartos, Yucatán, México. Mesoamericana, 15(2), 109. 
Cerezo, A., Robbins, C., \& Dowell, B. (2009). Uso de hábitats modificados por aves dependientes de bosque tropical en la región caribeña de Guatemala. Revista de Biología Tropical, 57(1-2), 401-419. DOI: 10.15517/rbt.v57i1-2.11355

Clarke, K. R. (1993). Non-parametric multivariate analyses of changes in community structure. Austral Ecology, 18(1), 117143. DOI: 10.1111/j.1442-9993.1993.tb00438.x

Daily, G. C., Ehrlich, P., \& Sánchez-Azofeifa. (2001). Countryside biogeography: use of human-dominated habitats by avifauna of southern Costa Rica. Ecological Application, 11, 1-3. DOI: 10.1890/10510761(2001)011[0001:CBUOHD]2.0.CO;2

Escudero, A., \& Rubio, A. (1994). Una breve revisión de técnicas de análisis multivariantes aplicables en Fitosociología. Botánica Complutensis, 19, 9-38.

González-Salazar, C., Martínez-Meyer, E., \& López-Santiago, G. (2014). A hierarchical classification of trophic guilds for North American birds and mammals. Revista Mexicana de Biodiversidad, 85, 931-941. DOI: 10.7550/rmb.38023

Gysel, L., \& Lyon. L. (1990). Análisis y evaluación de hábitat. In R. Rodríguez (Ed.), Manejo y gestión de la vida silvestre (pp. 321-344). USA: WWF/Fish and Wildlife Service.

Harvey, C. A., Medina, A., Sánchez, D. M., Vilcehz, S., Hernández, B., Saenz, J. C., ... Sinclair, F. L. (2006). Patterns of animal diversity in different forms of tree cover in agricultural landscapes. Ecological Applications, 16, 1986-1999. DOI: 10.1890/1051-0761(2006)016[1986:POADID]2.0.CO;2

Herrando-Pérez, S. (2002). Manual de ecología matemática: Un enfoque práctico al análisis multivariado (PCA, Cluster y MDS) para detectar patrones en ecología. Quintana Roo, México: ECOSUR-Chetumal.

Holdridge, L. R. (1970). Life zone ecology. San José, Costa Rica: Tropical Science Center.

Idropo-Medina, C., \& Gallo-Cajiao, E. (2012). Movilidad de aves de sotobosque entre fragmentos de bosque subandino en los Andes colombianos. Memorias Manejo de Fauna Silvestre en Amazonia y Latinoamérica. Recuperado de http://www.comfauna.org/wp-

content/uploads/2012/PDFsManejofaunasilvestre/Iquitos2004/3_Investigacion_Biologica_Aplicable_al_Manejo/ 195- 202_cidrobo_MovilidadAvesSotobosqueFragmentosBosque.pdf

Jiménez, A. (2013). Caracterización de la avifauna terrestre de la Reserva Natural Absoluta Cabo Blanco, sector Cabuya. Puntarenas, Costa Rica (Informe final de Práctica Dirigida para optar por el título de Bachiller en Manejo de Recursos Naturales). Universidad Estatal a Distancia, San José, Costa Rica.

Kricher, J. C. (1999). A neotropical companion. United Kingdom: Princeton University Press.

Lambin, E. F., Geits, H. J., \& Lepers, E. (2003). Dynamics and land-use and land-cover change in tropical regions. Annual Review of Environment and Resources, 28, 205-241. DOI: 10.1146/annurev.energy.28.050302.105459

Leighton, J., Mendenhall, C., Rosales, A., Zahawi, R., \& Holl, K. (2014). Landscape context mediates avian habitat choice in tropical forest restoration. Plos one, 9(3), 1-8. DOI: 10.1371/journal.pone.0090573

Loiselle, B. A. (1988). Bird abundance and seasonality in a Costa Rican lowland forest canopy. The Condor, 90, 761-772. DOI: $10.2307 / 1368833$

Luck, G. W., \& Daily, G. C. (2003). Tropical countryside bird assemblages: richness, composition, and foraging differ by landscape context. Ecological Applications, 13(1), 235-247. DOI: 10.1890/10510761(2003)013[0235:TCBARC]2.0.CO;2

MacArthur, R. H., \& Wilson, E. O. (1967). The theory of island biogeography. Princeton, USA: Princeton University Press.

Marini, M. A. (2001). Effets of forest fragmentation on birds of the Cerrado Region, Brazil. BirdConservation International, 11, 13-25. DOI: 10.1017/S0959270901001034 
Martínez-Salinas, A., \& DeClerck, F. (2010). The role of agroecosystems in the conservation of birds with in biological corridors. Mesoamericana, 14, 35-50.

Meave, J. A., Romero-Romero, M. A., Salas-Morales, S. H., Pérez-García, E. A., \& Gallardo-Cruz, J. A. (2012). Diversidad, amenazas y oportunidades para la conservación del bosque tropical caducifolio en el estado de Oaxaca, México. Ecosistemas, 21 (1-2), 85-100.

Naranjo, L. \& Chacón, P. (1997). Diversidad de insectos y aves insectívoras de sotobosque en hábitats perturbados de selva Iluviosa tropical. Caldasia, 19(3), 507-520.

Oksanen, J. (2015). Multivariate analysis of ecological communities in $R$ : vegan tutorial. Recuperado de http://cc.oulu.fi/ jarioksa/opetus/metodi/vegantutor.pdf

Oksanen, J., Blanchet, F. G., Kindt, R., Legendre, P., Michin, P. R., O’Hara, R. B., ... Wagner, E. (2013). Community Ecology Package (Packagevegan). Recuperado de http://vegan.r-forge.r-project.org/

Oviedo, P. E. (2015). Composición y estructura forestal de la Reserva Natural Absoluta de Cabo Blanco. Repertorio Científico, 18(2), 95-102.

Petit, L. J., \& Petit, D. R. (2003). Evaluating the importance of human-modified lands for Neotropical bird conservation. Conservation Biology, 17, 687-694. DOI: 10.1046/j.1523-1739.2003.00124.x

Pimm, S. L., \& Raven, P. (2000). Extinction by numbers. Nature, 403, 843-845. DOI: 10.1038/35002708

Quesada, R. (2007). Los bosques de Costa Rica (IX Congreso Nacional de Ciencias). Cartago, CR: Instituto Tecnológico de Costa Rica.

Ralph, C. J., Geupel, G. R., Pyle, P., Martin, T. E., DeSante, D., \& Milá, B. (1996). Manual de métodos de campo para el monitoreo de aves terrestres. California, USA: General TechnicalReport PSW-GTR-159. DOI: 10.2737/PSW-GTR-159

Rangel-Salazar, J., Enríquez, P., \& Sántiz, E. (2009). Variación de la diversidad de aves de sotobosque en el parque nacional Lagos de Montebello, Chiapas, México. Acta ZoológicaMexicana, 25(3), 479-495. DOI: 10.21829/azm.2009.253655

Rappole, J. H., \& Morton, E. S. (1985). Effects of habitat alteration on a tropical avian forest community. In P. A. Buckley (Ed), Neotropical Ornithology (pp. 1013-1020). Washington, D.C, USA: The American Ornithologist Union. DOI: $10.2307 / 40168333$

Sáenz, J. C., Villatoro, F., Ibrahim, M., Fajardo, D., \& Pérez, M. (2006). Relación entre las comunidades de aves y la vegetación en agropaisajes dominados por la ganadería en Costa Rica, Nicaragua y Colombia. Agroforestería en las Américas, 45, 37-48.

Sánchez-Ulate, N. (2011). Abundancia de tres especies de aves insectívoras de sotobosque y disponibilidad de recursos en bosques tropicales de Ilanura, Sarapiquí, Costa Rica (Tesis Magister Scientiae en Conservación y Manejo de Vida Silvestre). Universidad Nacional, Instituto Internacional en Conservación y Manejo de Vida Silvestre, Heredia, Costa Rica.

Sekercioglu, C. (2002). Effects of forestry practices on vegetation structure and bird community of Kibale National Park, Uganda. Biological Conservation, 107, 229-240. DOI: 10.1016/S0006-3207(02)00097-6

Sekercioglu, C. H., Ehrlich, P. R., Daily, G. C., Argen, D., Goehring, D., \& Sandi, R. (2002). Dissapearance of insectivorous birds from tropical forest fragments. Proceedings of the National Academy of Sciences, 99, 263-267. DOI: 10.1073/pnas.012616199

Stiles F. G. (1985). Conservation of forest birds of Costa Rica: problems and perspectives In A. W. Diamond \& T. S. Lovejoy (Eds.), Conservation of tropical forest birds (Technical Publication Number 4) (pp. 121-138). Cambridge, USA: International Council for Bird Preservation.

Stiles, F. G., \& Skutch, A. (2003). Guía de aves de Costa Rica. Heredia, Costa Rica: INBio. 
Stotz, D. F., Fitzpatrick, J. W., Parker, T. A., \& Moskovits, D. K. (1996). Neotropical Birds: Ecology and Conservation. Chigago, EE. UU: The University of Chicago Press.

Stratford, J. A., \& Stouffer, P. C. (1999). Local extinctions of terrestrial insectivorous birds in fragmented landscape near Manaus, Brazil. Conservation Biology, 13(6), 1416-1423. DOI: 10.1046/j.1523-1739.1999.98494.x

Verea, C., \& Solorzano A. (1998). La avifauna del sotobosque de una selva decidua tropical en Venezuela. Ornitología Neotropical, 9, 167-176.

Villegas, J. C., \& Villalobos, J. (2014). Avifauna del Caribe sur asociada a ecosistemas alterados en Limón, Costa Rica. Cuadernos de Investigación, 6(2), 187-196. DOI: 10.22458/urj.v6i2.623

Weins, J. A., \& Rotenberry, J. T. (1981). Habitat associations and community structure of birds in shrub steppe. Ecological Monographs, 51(1), 21-42. DOI: 10.2307/2937305 